\title{
Easy quantitative methodology to assess visual-motor skills
}

This article was published in the following Dove Press journal:

Neuropsychiatric Disease and Treatment

II January 2013

Number of times this article has been viewed

\author{
Matteo Chiappedi' \\ Alessio Toraldo ${ }^{2}$ \\ Silvia Mandrini ${ }^{3}$ \\ Federica Scarpina ${ }^{2}$ \\ Melissa Aquino ${ }^{2}$ \\ Francesca Giulia Magnani \\ Maurizio Bejor ${ }^{3}$ \\ 'Don Carlo Gnocchi ONLUS \\ Foundation, Milan, Italy; ${ }^{2}$ University \\ of Pavia, Department of Psychology, \\ Pavia, Italy; ${ }^{3}$ University of Pavia, \\ Department of Surgical, Resuscitative, \\ Rehabilitative and Transplant Sciences, \\ Pavia, Italy
}

Introduction: Visual-motor skills are the basis for a great number of daily activities. To define a correct rehabilitation program for neurological patients who have impairment in these skills, there is a need for simple and cost-effective tools to determine which of the visual-motor system levels of organization are compromised by neurological lesions. In their 1995 book, The Visual Brain in Action (Oxford: Oxford University Press), AD Milner and MA Goodale proposed the existence of two pathways for the processing of visual information, the "ventral stream" and "dorsal stream," that interact in movement planning and programming. Beginning with this model, our study aimed to validate a method to quantify the role of the ventral and dorsal streams in perceptual and visual-motor skills.

Subjects and methods: Nineteen right-handed healthy subjects (mean age 22.8 years \pm 3.18 ) with normal or corrected-to-normal vision were recruited. We proposed that a delayed pointing task, a distance reproduction task, and a delayed anti-pointing task could be used to assess the ventral stream, while the dorsal stream could be evaluated with a grasping task and an immediate pointing task. Performance was recorded and processed with the video-analysis software Dartfish ProSuite.

Results: Results showed the expected pattern of predominance of attention for the superior left visual field, predominance of the flexor tone in proximal peri-personal space arm movements, tendency toward overestimation of short distances, and underestimation of long distances.

Conclusion: We believe that our method is advantageous as it is simple and easily transported, but needs further testing in neurologically compromised patients.

Keywords: dorsal stream, ventral stream, visual-motor skills, rehabilitation, neurological disorders

\section{Introduction}

Visual-motor skills are essential for the dexterity of the upper extremities ${ }^{1}$ and for a wide range of daily activities involving movements of object achievement (reaching) and gripping (grasping), where vision guides the motor action. ${ }^{2}$

Hand-eye coordination can be described as an ordered sequence consisting of: visualization of the target, focus of attention on it, identification and location of the target, planning and programming of the movement, activation of the upper limb muscles to start and perform the movement, and control of the action performed. ${ }^{3}$ These processes require the integration of different systems, including the somatosensory and perceptual system, central processing systems (especially those involved in monitoring, controlling, attention, and motivation), and the motor system. During these phases, visual and proprioceptive information is integrated to drive and support
Correspondence: Matteo Chiapped Rehabilitation Unit, Santa Maria alle Fonti Medical Center, Don Carlo Gnocchi ONLUS Foundation, Viale Mangiagalli 52, 27052 Salice Terme, Italy

Tel +390383945612

$\mathrm{Fax}+390383945678$

Email mchiappedi@dongnocchi.it 
the movement of the upper limb, providing feed-back and feed-forward control. ${ }^{4,5}$

Given the importance of visual-motor skills in activities of daily living, the significance of their impairment can easily be understood. Such impairment is a rather common result of central nervous system damage. The proper planning of the rehabilitative treatment of these patients requires the development and application of assessment systems that are able to determine which of the organization levels of the visual-motor system are compromised and to quantify the results of intervention. ${ }^{6}$

Information received in the visual areas of the occipital lobe is projected to several areas in the visual associative parietal and temporal lobes. Ungerleider and Mishkin identified, based on previous theories and experimental data obtained from cortical lesions of the cortex of monkeys, the presence of two pathways from the primary visual cortex to higher centers. ${ }^{7}$ The first was called "dorsal stream" for its location in the dorsal convexity of brain cortex; it connects the primary visual area (V1) to the posterior parietal lobe. It was considered responsible for the localization of objects (the "where" stream). The second was called the "ventral stream"; it is centered on the V4 area and connects the V1 area to the inferior temporal lobe (ITL). It was considered responsible for the recognition of objects (the "what" stream). ${ }^{8}$

Goodale and Milner later proposed a new interpretation of the fundamental organization of the visual system. Studying their patient with bilateral lesions in the occipito-lateral lobes and a small lesion in the left posterior parietal cortex, but with completely spared visual areas, ${ }^{9}$ they showed a profound deficit in shape discrimination, but retained reaching ability. ${ }^{10}$ Studies in healthy subjects also showed their ability, under certain experimental conditions, to act without being conscious of their action. ${ }^{11}$ On the basis of such experimental evidence, Milner and Goodale have suggested that the fundamental difference between the two pathways is not in terms of percept type (space vs object) but in terms of the use of the information in the higher centers (action vs perception/recognition). ${ }^{12}$

The ventral stream carries information necessary for the perception and recognition of the stimulus, while the dorsal stream carries that information required for controlling movements directed to the stimulus. A rather similar proposal had been advanced by Jeannerod, who suggested distinguishing a "semantic" from a "pragmatic" way of processing information for, respectively, the conscious understanding of the outside world and motor programming. ${ }^{13}$
The dorsal stream carries the visual information concerning the position of objects moment by moment to coordinate limb movements in the visual space. Thus, it is more involved in the control and integration of stimuli in peripheral and lower visual fields, where hands, feet, and the ground are usually found. ${ }^{14}$ The overexpression of lower visual fields in the dorsal stream is evident, for example, in the V6A area: it has been shown that pointing movements are faster and more accurate when they are carried out in the lower visual fields, compared with when the same movements are carried out in the upper visual field. ${ }^{15}$

A functional deficit of the dorsal stream is responsible for optic ataxia, characterized by errors in the direction of reaching movements of the hand toward an object of interest, especially when this is placed in the periphery of the visual field. ${ }^{16,17}$ A lesion of the ventral stream can produce visual agnosia, in which the recognition of objects is not possible through visual information, and unilateral spatial neglect (neglect syndrome), in which the patient tends to ignore stimuli in the visual field opposite to the lesion.

The ventral and dorsal streams are not independent but functionally dissociated. The two streams are activated differently if the action is directed to a visible target or to one that has not been visible in the last 2 or more seconds. ${ }^{18}$ Milner et al have shown that a patient with lesions of the dorsal stream and optic ataxia, improved their performance in pointing tasks when the stimulus disappeared more than 2 seconds before the start of the movement. ${ }^{19}$

The objective of the study reported here was the validation of a method able to quantify the perceptual (ie, ventral stream) and visual-motor (ie, dorsal stream) skills in a sample of healthy subjects, to obtain a range of normality values. We expected that the obtained quantifications would confirm the functional patterns already described in scientific literature.

\section{Subjects and methods}

The study was performed in May 2012 in the Rehabilitation Unit of the Santa Maria alle Fonti Medical Center, part of the Don Carlo Gnocchi ONLUS Foundation.

\section{Study sample}

We recruited 19 right-handed healthy adults with normal or corrected vision (females:males, 14:5; age 22.8 \pm 3.18 years; education $15.78 \pm 1.35$ years). All subjects were either students or residents at the University of Pavia. The exclusion criteria of this study were a history of psychiatric illness, anamnesis or actual alcohol abuse, recent trauma involving the 
upper arm, and/or visual deficits not adequately compensated for by binocular lenses. All subjects were informed about the procedures and purposes of the investigation and they signed written informed consent. The study was conducted following recommendations from the Helsinki Declaration.

\section{Experimental tasks}

\section{Assessment of the ventral stream}

Each subject sat at a table covered with a non-reflective material to avoid reflections during the tests and to ensure clear video capture. A 22-inch LCD screen was used for the presentation of visual stimuli. This was placed on the table in front of the subject, at approximately 55-60 centimeters distance from the subject's eyes. A special lighting system set at 100 watts was used and the point on which people had to rest the index finger of their right hand during projection of the stimulus and where they had to return at the end of each trial was clearly marked (Figure 1).

The subject could not see their own right hand during stimulus presentation because the working space was covered; the cover was removed 2 seconds after the stimulus disappeared from the screen. The subject, who was given unlimited time for visual analysis, controlled the disappearing of the stimulus.

For each trial, the target stimulus consisted of a black circle ( $5 \mathrm{~mm}$ diameter) projected on a white background. This stimulus was positioned at distances of $3,6,9 \mathrm{~cm}$ and along rays at 45, 135, 180, 225, 270, and 315 degrees compared with a black cross $(5 \times 5 \mathrm{~mm})$ located centrally in the field of projection.

Each subject was preliminarily allowed to undertake a number of trials of each test sufficient to make sure that they understood the task exactly. These trials were not recorded.

In the delayed pointing $(\mathrm{P})$ task, the visual stimulus was presented and, after 2 seconds without visual input, the subject was asked to indicate with their index finger the target position with respect to the point marked on the shelf (the starting point; see Figure 2). In the delayed distance (D) task, after the presentation of the stimulus and a latency period of two seconds, the subject was asked to mimic with the first and second fingers the distance between the two elements.

The two tasks were administered in separate blocks following the ABBA order. The positions along the horizontal axis, 0 and 180 degrees, were presented three times because they were thought to be the most significant positions; ${ }^{20}$ therefore, for each block there were 36 trials, 18 of which related to non-horizontal positions $(45,90,135,225,270$, or 315 degrees) and 18 related to horizontal positions $(0,180$ degrees). Pseudo-randomized sequences were used to avoid the construction of sequences with identical consecutive stimuli and sequences with more than three consecutive stimuli on the horizontal axis.

In the horizontal delayed pointing (DP) task, we asked the subjects to indicate with their index finger the remembered position of the visual target; in the horizontal delayed anti-pointing (DAP) task, the subjects instead had to indicate the symmetrical (with respect to the starting point) position. Both tasks were performed 2 seconds after the stimulus disappeared. Since the horizontal axis is the most sensitive in the detection of dysfunction after brain injury, we presented stimuli at distances of 3,6 , or $9 \mathrm{~cm}$ from the central cross on the 0 and 180 degrees axis, for a total of 18 trials per task.

\section{Assessment of the dorsal stream}

The visual-motor skills relating to the dorsal stream were evaluated with tests in which the action (reaching or grasping) was executed without any latency and while the visual stimulus remained visible.

In the immediate pointing (IP) task, a black circle $(5 \mathrm{~mm}$ diameter) and a central cross $(5 \times 5 \mathrm{~mm})$ were printed on A4 white paper. The target stimulus (black circle) was presented at distances of 3,6 , or $9 \mathrm{~cm}$ along the rays of 45, 135, 180, 225,270 , and 315 degrees with respect to the central cross. We asked the subject to indicate the black circle. A total of 36 trials were administered, with the stimuli presented in random order.

In the grasping $(\mathrm{G})$ task, gray rulers of $1 \times 1 \times 3 \mathrm{~cm}$, $1 \times 1 \times 6 \mathrm{~cm}$, and $1 \times 1 \times 9 \mathrm{~cm}$ size were inclined 45 or 90 degrees to the axis of symmetry of an A4 white sheet of paper. We asked the subject to grasp the ends of the rulers with their first and second fingers. A total of 36 trials were administered, with the different rulers and positions presented in random order.

\section{Detection apparatus}

A camera orthogonally placed at about $50 \mathrm{~cm}$ from the plane filmed all movements. The videos were reworked with the video analysis software Dartfish ProSuite 5.0 (Dartfish, Fribourg, Switzerland). This software allowed measurement of the positions and distances pointed by the subjects (see Figure 3). Data obtained were then transferred to a spreadsheet for statistical analysis.

At the beginning of each new task administration, we calibrated our system by filming a ruler with millimeter 

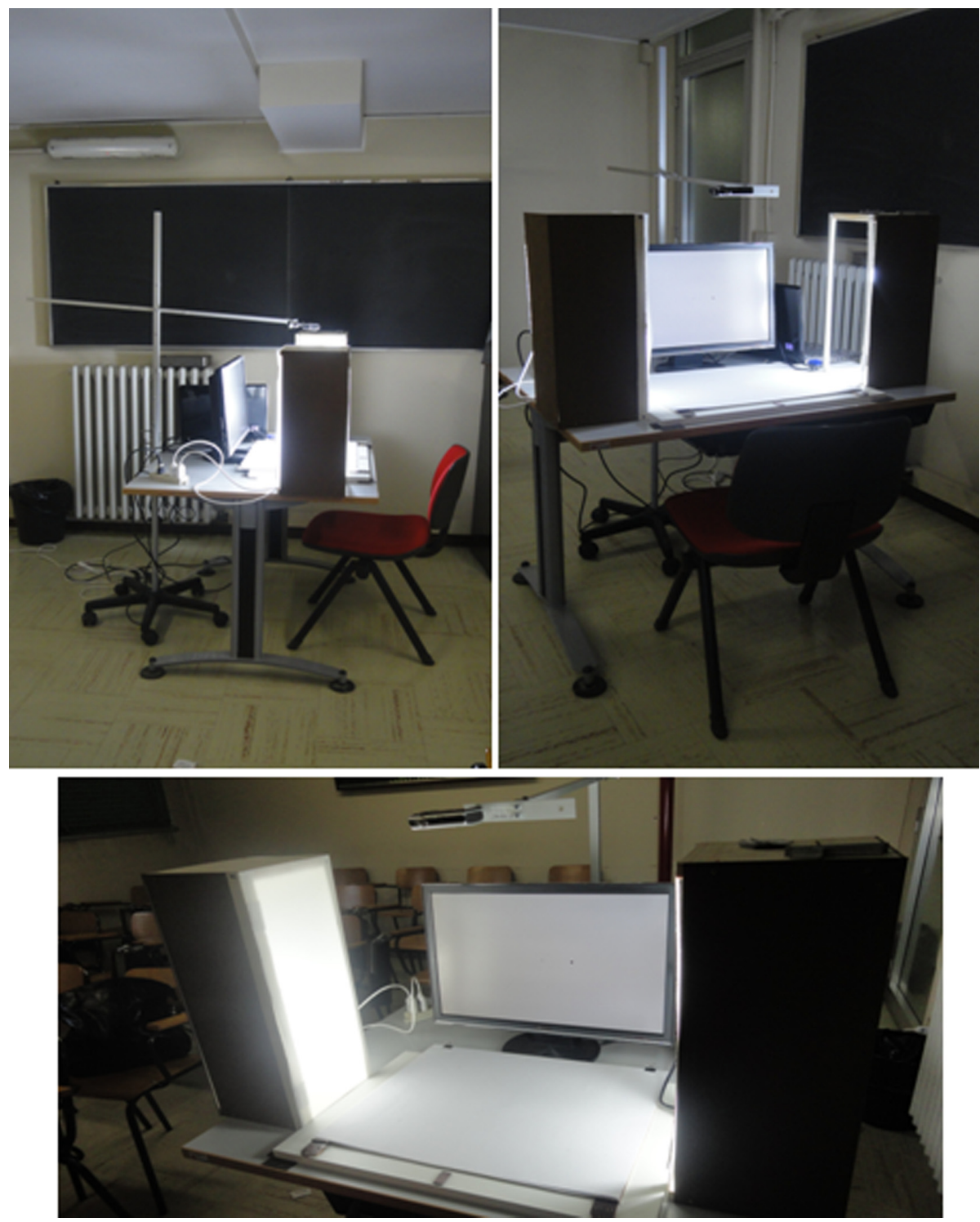

Figure I The setting.

increments marked on it placed on the shelf, to correct any possible distortion in the recorded video.

\section{Statistical analysis}

Data were analyzed with SPSS (v 19; IBM, Armonk, New York, USA).

For the P, D, IP, DP, and DAP tasks, we calculated the differences between the real distances and those indicated by each subject. For the $\mathrm{G}$ task, we calculated the differences between the maximum opening of the first and second finger before the ruler was grasped and the ruler's length. Therefore, in the first group of mentioned tasks, we quantified measure errors, while, for the $G$ task, we measured the excess of distance between the first phalanges of the first and second finger at about two-thirds of the flight phase compared with the real size of the object to be grasped.

For the IP task, we considered the number of errors (defined as pointing in a place different from the location of the stimulus).

Each task was analyzed with a general linear model (GLM), which can be described as a generalization of a multiple linear regression model to more than one dependent variable. A difference was considered significant if $P \leq 0.05$.

\section{Results

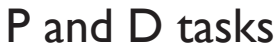

The means and standard deviations of the systematic error in the P and D tasks are shown in Table 1. 


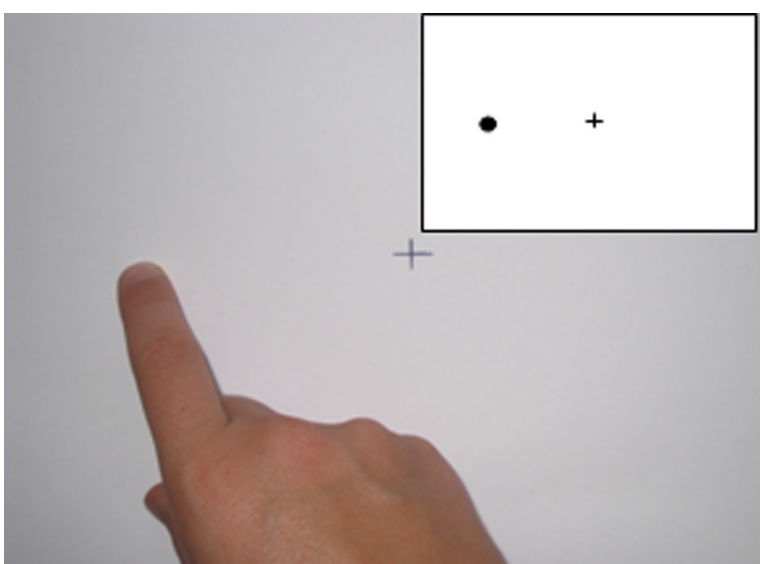

Figure 2 Performance in delayed pointing task (stimulus represented in box).

We divided data in groups according to the target position on the visual fields (both on the horizontal and vertical planes).

We first applied a GLM $2(\mathrm{P}$ or $\mathrm{D}) \times 3$ (spatial position: left, center, right). Data analysis revealed a significant interaction between the stimuli spatial position and the task $[\mathrm{F}(1.336,0.856)=4.53 ; P=0.017]$. We highlighted a linear effect of stimuli position on the task $[\mathrm{F}(1,1.625)=5.914$; $P=0.026]$ in the $\mathrm{P}$ task but not in the $\mathrm{D}$ task.

We also applied a GLM $2(\mathrm{P}$ or D) $\times 3$ (spatial position: superior, middle, inferior). We found a significant interaction between the position of the stimulus and the task $[\mathrm{F}(1.71$, $2.49)=32.03 ; P<0.001]$. In both the $\mathrm{P}$ and $\mathrm{D}$ tasks, we highlighted a linear effect of stimuli position on the task: $\mathrm{F}(1,0.477)=21.32(P<0.001)$ and $\mathrm{F}(1,0.377)=11.01$ $(P=0.004)$, respectively.

Means and standard deviations of the random error are shown in Table 2. No significant effects or interactions were found.

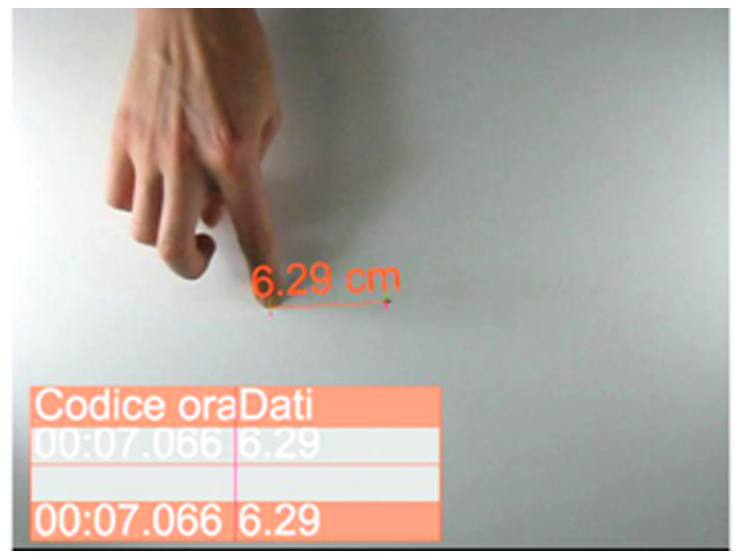

\section{$P$ and DAP tasks}

Means and standard deviations of the systematic error are shown in Table 3.

We applied a GLM $2($ P or DAP $) \times 2$ (reaction/response side: left or right) $\times 3$ (distance: 3,6 , or $9 \mathrm{~cm}$ ). The analysis revealed a significant effect of stimuli distance from the reference cross $[\mathrm{F}(1.07,28.12)=16.84 ; P=0.001]$ on both tasks. The means and standard deviations of random error are shown in Table 4.

We applied a GLM $2($ P or DAP $) \times 2$ (reaction/response side: left or right) $\times 3$ (distance: 3,6 , or $9 \mathrm{~cm})$. Data analysis revealed a significant effect of the distance of the stimulus from the reference cross $[\mathrm{F}(1.78,2.37)=25.8 ; P<0.001]$ on both tasks: subjects overestimated short distances and underestimated long distances. Presentation side of the stimulus did not seem to influence task performance.

\section{G task}

Means and standard deviations of the systematic error are shown in Table 5.

We applied a GLM 2 (ruler inclination: 45 or 90 degrees) $\times 3$ (ruler length: 3,6 , or $9 \mathrm{~cm}$ ). Data analysis showed a significant effect of both ruler length $[\mathrm{F}(1.53$, $145.26)=733.30 ; P<0.001]$ and inclination $[\mathrm{F}(1$, $238.58)=1707.22 ; P<0.001]$, without any statistically significant interaction between the two.

Subjects opened their fingers about $20 \%$ more than necessary according to ruler length, a percentage that tended to increase when the ruler was placed at 45 degrees.

\section{IP task}

Statistical analysis was not conducted because subjects made no errors during this task.

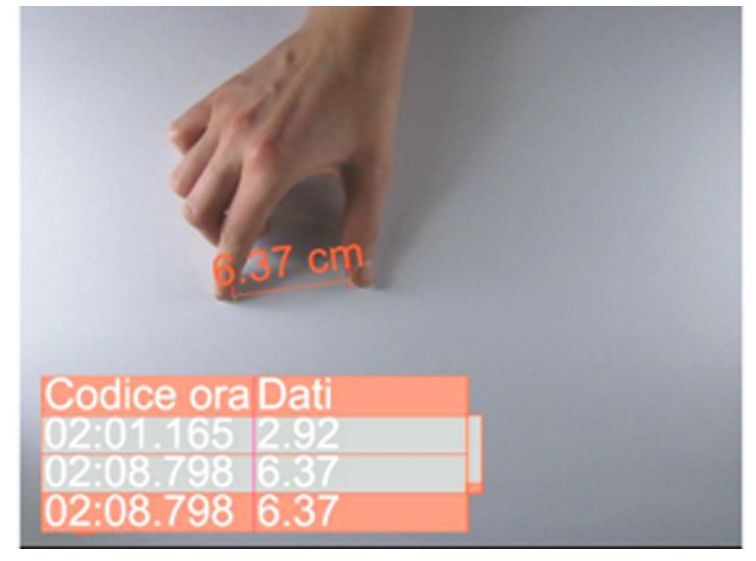

Figure 3 Data resulting from video analysis in a pointing and in a grasping task.

Notes: Codice ora is Italian for Time (measured from the beginning of the task) and Dati is Italian for Measure (in centimeters). 
Table I Systematic error in delayed pointing and delayed distance tasks (mean [standard deviation])

\begin{tabular}{lll}
\hline Presented & Delayed pointing & Delayed distance \\
\hline Left & $0.33(0.80)$ & $0.59(1.14)$ \\
Center & $0.46(0.65)$ & $0.24(0.95)$ \\
Right & $0.34(0.57)$ & $0.11(0.77)$ \\
Inferior & $0.73(0.89)$ & $0.24(0.89)$ \\
Middle & $0.41(0.55)$ & $0.13(0.87)$ \\
Superior & $0.02(0.67)$ & $0.43(0.91)$ \\
\hline
\end{tabular}

\section{Discussion}

For tasks studying the ventral stream, conditions were chosen to avoid the activation of the dorsal stream. We used three study conditions to achieve this goal. First, the answer to the task was given on a plane that was orthogonal to the plane where the stimulus had been seen. This was done to force subjects to activate the streams and not to rely on a simple memory of the position of the stimulus. Second, we used a latency of 2 seconds, as Milner and Goodale have suggested is a time that enhances activation of the dorsal stream over the ventral stream when performing a task. ${ }^{18,19}$ Third, a movable shelf was used to prevent the subject from watching their own hand while receiving the input in tasks meant to activate the ventral rather than the dorsal stream.

\section{$P$ and $D$ tasks}

In the $\mathrm{P}$ task, statistical analysis revealed that the systematic error tended to be higher for stimuli projected in the inferior part of the visual field. This could be explained by a predominance of the flexor tone occurring during arm pointing movements in proximal peri-personal space.

In contrast, in the D task, the error tended to be higher for stimuli projected in the superior part of the visual field. Brain lateralization could play a role in explaining this phenomenon: the right hemisphere of the brain is specialized in visuo-spatial representation and has been shown to perform a more careful analysis of stimuli projected in the superior left part of the visual field. ${ }^{22}$ Moreover, all subjects we tested were from a Western culture: the

Table 2 Random error in delayed pointing and delayed distance tasks (mean [standard deviation])

\begin{tabular}{lll}
\hline Presented & Delayed pointing & Delayed distance \\
\hline Left & $1.04(0.74)$ & $0.91(0.26)$ \\
Center & $1.14(0.47)$ & $0.82(0.32)$ \\
Right & $1.06(0.67)$ & $0.79(0.35)$ \\
Inferior & $0.96(0.62)$ & $0.88(0.49)$ \\
Middle & $1.01(0.79)$ & $0.83(0.36)$ \\
Superior & $0.98(0.54)$ & $0.96(0.52)$ \\
\hline
\end{tabular}

Table 3 Systematic error in delayed pointing and delayed anti-pointing tasks (mean [standard deviation])

\begin{tabular}{lll}
\hline Length & Delayed pointing & Delayed anti-pointing \\
\hline $3 \mathrm{~cm}$ & $0.68(0.58)$ & $0.77(0.56)$ \\
$6 \mathrm{~cm}$ & $0.17(0.68)$ & $0.44(0.59)$ \\
$9 \mathrm{~cm}$ & $-0.21(0.82)$ & $-0.22(0.93)$ \\
\hline
\end{tabular}

habit of reading from left to right could contribute to the measured differences.

\section{$P$ and DAP tasks}

In these tasks, the only factor influencing performance seems to have been target distance from the reference cross. We registered an overestimation (of about $0.75 \mathrm{~cm}$ ) for a short distance $(3 \mathrm{~cm}$ ) and an underestimation (of about $-0.20 \mathrm{~cm}$ ) for a long distance $(9 \mathrm{~cm})$. To explain this, it could be hypothesized that an unconscious "obstacle avoidance behavior" intervened. ${ }^{21}$ According to this hypothesis, the reference cross would be perceived as an "obstacle" and subjects would overestimate the target position when it was close to the reference cross to avoid it. This behavior would prevail on the tendency toward an underestimation of target distance, which emerged when the stimulus was further away from the reference cross.

Statistical analysis of random error highlighted a significant interaction between distance and subject's performance: response variability was wider for longer distances, without a significant effect of the side of presentation of the stimulus.

\section{G task}

The excess of finger opening could be explained by neuromotor constraints: the shoulder, elbow, and wrist drawing movement near the body involve a predominance of flexor tone. In healthy subjects, this is balanced by the activation of antagonist extensor muscles. The predominance of arm muscle flexor tone is greater when the ruler reaches an inclination of 45 degrees rather than 90 degrees; to counteract this, subjects tend to increase the activation of the extensor muscles, including those acting on the fingers.

Table 4 Random error in delayed pointing and delayed antipointing tasks (mean [standard deviation])

\begin{tabular}{lll}
\hline Length & Delayed pointing & Delayed anti-pointing \\
\hline $3 \mathrm{~cm}$ & $0.45(0.54)$ & $0.43(0.43)$ \\
$6 \mathrm{~cm}$ & $0.65(0.3 \mathrm{I})$ & $0.60(0.40)$ \\
$9 \mathrm{~cm}$ & $0.82(0.4 \mathrm{I})$ & $0.75(0.55)$ \\
\hline
\end{tabular}


Table 5 Systematic error in grasping task (mean [standard deviation])

\begin{tabular}{llll}
\hline & $3 \mathbf{~ c m}$ ruler & $\mathbf{6} \mathbf{c m}$ ruler & $\mathbf{9} \mathbf{c m}$ ruler \\
\hline 45 degrees & $3.91(0.44)$ & $6.02(0.63)$ & $7.67(0.56)$ \\
90 degrees & $1.00(0.39)$ & $2.96(0.48)$ & $4.48(0.46)$ \\
\hline
\end{tabular}

Moreover, wrist position influences the finger extrinsic muscles: at an inclination of 45 degrees, the activation of the forearm extensor muscles is stronger and this leads to an increased contrast of the action of the flexor digitorum profundus, flexor digitorum superficialis and flexor pollicis longus. This could result in a relative reduction in the flexor tone acting on the fingers.

\section{IP task}

The IP task was designed to investigate visual-motor skills depending on the dorsal stream and to detect the presence of pathological conditions such as optic ataxia. As expected for a healthy sample, all performances were completely normal.

\section{Conclusion}

Our method provides findings in line with the published literature. Accuracy and precision increased when pointing targets were located in the superior left visual field. ${ }^{22}$ A predominance of the flexor tone in proximal peri-personal space arm movements was evident. ${ }^{23}$

The absence of significant random errors in any task and the accuracy in data collection are significant factors increasing the validity of our method. The innovative technique we explored allowed us to obtain easily precise measurement of finger position, distances, and aperture of finger grip. It is not an invasive procedure and does not require the use of fixed markers. Thus, we believe that our method could be useful in many settings, because it is as easy to reproduce as the commonly used paper and pencil tests and does not cost as much as more complex devices. ${ }^{6}$

However, our study has some relevant limitations. The major limitation is that we recruited only healthy subjects; as such, we need to collect data from patients known to have a disruption of the dorsal and/or ventral stream due to a neurological disorder. This is the only way to confirm whether our tasks can discriminate between these conditions. Moreover, we will need to perform the same assessment before and after treatment to verify the tasks' sensitivity to change.

Future studies need to be conducted with a comparison assessment technique to verify the reliability of our method.

\section{Disclosure}

The authors received no grants or funding for this paper and declare no conflicts of interest in this work.

\section{References}

1. Desrosiers J, Hébert R, Bravo G, Dutil E. Upper-extremity motor coordination of healthy elderly people. Age Ageing. 1995;24(2):108-112.

2. Crawford JD, Medendorp WP, Marotta JJ. Spatial transformations for eye-hand coordination. J Neurophysiol. 2004;92(1):10-19.

3. Jeannerod M. Mechanisms of visuomotor coordination: a study in normal and brain-damaged subjects. Neuropsychologia. 1986;24(1):41-78.

4. Turvey MT, Fonseca S. Nature of motor control: perspective and issues. In: Sternad D, editor. Progress in Motor Control: A Multidisciplinary Perspective. New York, NY: Springer Verlag; 2009:93-123.

5. Gottlieb GL, Song Q, Almeida GL, Hong DA, Corcos D. Directional control of planar human arm movement. J Neurophysiol. 1997;78(6): 2985-2988.

6. Chiappedi M, De Bernardi E, Dalla Toffola E, Bejor M. Child visuomotor skills: preliminary findings using a new low-cost movement analysis method. Funct Neurol. 2010;25(1):45-48.

7. Ungerleider L, Mishkin M. Two cortical visual systems. In: Ingle DJ, Goodale MA, Mansfield RJW, editors. Analysis of Visual Behaviour. Cambridge, MA: MIT Press; 1982:549-586.

8. Rizzolati G, Sinigaglia C. So quel che fai. Il cervello che agisce e $i$ neuroni specchio [I know what you're doing: the acting brain and mirror neurons]. Milan: Raffaello Cortina Editore; 2006.

9. James TW, Culham J, Humphrey GK, Milner AD, Goodale MA. Ventral occipital lesions impair object recognition but not object-directed grasping: an fMRI study. Brain. 2003;126(Pt 11):2463-2475.

10. Milner AD, Perrett DI, Johnston RS, et al. Perception and action in 'visual form agnosia'. Brain. 1991;114(Pt 1B):405-428.

11. Dehaene S, Changeux JP, Naccache L, Sackur J, Sergent C. Conscious, preconscious, and subliminal processing: a testable taxonomy. Trends Cogn Sci. 2006;10(5):204-211.

12. Milner AD, Goodale MA. The Visual Brain in Action. Oxford: Oxford University Press; 1995.

13. Jeannerod M. The representing brain: neural correlates of motor intention and imagery. Behav Brain Sci. 1994;17(2):187-202.

14. Motter BC, Mountcastle VB. The functional properties of the lightsensitive neurons of the posterior parietal cortex studied in waking monkeys: foveal sparing and opponent vector organization. J Neurosci. 1981;1(1):3-26.

15. Danckert J, Goodale MA. Superior performance for visually guided pointing in the lower visual field. Exp Brain Res. 2001;137(3-4):303-308.

16. Gréa H, Pisella L, Rossetti Y, et al. A lesion of the posterior parietal cortex disrupts on-line adjustments during aiming movements. Neuropsychologia. 2002;40(13):2471-2480.

17. Pisella L, Gréa H, Tilikete C, et al. An 'automatic pilot' for the hand in human posterior parietal cortex: toward reinterpreting optic ataxia. Nat Neurosci. 2000;3(7):729-736.

18. Westwood DA, Goodale MA. Perceptual illusion and the real-time control of action. Spat Vis. 2003;16(3-4):243-254.

19. Milner AD, Paulignan Y, Dijkerman HC, Michel F, Jeannerod M. A paradoxical improvement of misreaching in optic ataxia: new evidence for two separate neural systems for visual localization. Proc Biol Sci. 1999;266(1434):2225-2229.

20. Lepelley MC, Thullier F, Bolmont B, Lestienne FG. Age-related differences in sensorimotor representation of space in drawing by hand. Clin Neurophysiol. 2010;121(11):1890-1897.

21. Milner AD, Goodale MA. Two visual systems re-viewed. Neuropsychologia. 2008;46(3):774-785.

22. de Montalembert M, Auclair L, Mamassian P. "Where is the sun" for hemi-neglect patients? Brain Cogn. 2010;72(2):264-270.

23. Kirsch W, Herbort O, Butz MV, Kunde W. Influence of motor planning on distance perception within the peripersonal space. PLoS One. 2012; 7(4):e34880 


\section{Publish your work in this journal}

Neuropsychiatric Disease and Treatment is an international, peerreviewed journal of clinical therapeutics and pharmacology focusing on concise rapid reporting of clinical or pre-clinical studies on a range of neuropsychiatric and neurological disorders. This journal is indexed on PubMed Central, the 'PsycINFO' database and CAS.

The manuscript management system is completely online and includes a very quick and fair peer-review system, which is all easy to use. Visit http://www.dovepress.com/testimonials.php to read real quotes from published authors.

\footnotetext{
Submit your manuscript here: http://www.dovepress.com/neuropsychiatric-disease-and-treatment-journal
} 